Corrigendum

\title{
Corrigendum to "TRIMAGE: A dedicated trimodality (PET/MR/EEG) imaging tool for schizophrenia” [Eur Psychiatry 50 (2018) 7-20]
}

\author{
Alberto Del Guerra ${ }^{\mathrm{a}, *}$, Salleh Ahmad ${ }^{\mathrm{b}}$, Mihai Avram $^{\mathrm{c}, \mathrm{d}, \mathrm{e}}$, Nicola Belcari $^{\mathrm{a}}$, Arne Berneking $^{\mathrm{f}}$, \\ Laura Biagi $^{g}$, Maria Giuseppina Bisogni ${ }^{\mathrm{a}}$, Felix Brandl ${ }^{\mathrm{d}, \mathrm{e}}$, Jorge Cabello ${ }^{\mathrm{c}}$, \\ Niccolò Camarlinghi ${ }^{\mathrm{a}}$, Piergiorgio Cerello ${ }^{\mathrm{h}}$, Chang-Hoon Choi ${ }^{\mathrm{f}}$, Silvia Coli $^{\mathrm{h}}$, \\ Sabrina Colpo ${ }^{\mathrm{i}}$, Julien Fleury ${ }^{\mathrm{b}}$, Vito Gagliardi ${ }^{\mathrm{a}}$, Giuseppe Giraudo ${ }^{\mathrm{h}}$, Karsten Heekeren ${ }^{\mathrm{j}}$, \\ Wolfram Kawohl ${ }^{\mathrm{j}, \mathrm{k}}$, Theodora Kostou ${ }^{\mathrm{l}}$, Jean-Luc Lefaucheur ${ }^{\mathrm{m}}$, Christoph Lerche ${ }^{\mathrm{f}}$, \\ George Loudos ${ }^{1}$, Matteo Morrocchi ${ }^{\mathrm{a}}$, Julien Muller ${ }^{\mathrm{n}}$, Mona Mustafa ${ }^{\mathrm{c}}$, Irene Neuner ${ }^{\mathrm{f}, \mathrm{n}}$, \\ Panagiotis Papadimitroulas ${ }^{\mathrm{l}}$, Francesco Pennazio ${ }^{\mathrm{h}}$, Ravichandran Rajkumar ${ }^{\mathrm{f}, \mathrm{o}}$, \\ Cláudia Régio Brambilla ${ }^{\mathrm{f}}$, Julien Rivoire ${ }^{\mathrm{n}}$, Elena Rota Kops ${ }^{\mathrm{f}}$, Jürgen Scheins ${ }^{\mathrm{f}}$, \\ Rémy Schimpf ${ }^{\mathrm{n}}$, N. Jon Shah ${ }^{\mathrm{f}}$, Christian Sorg ${ }^{\mathrm{d}, \mathrm{e}, \mathrm{q}}$, Giancarlo Sportelli ${ }^{\mathrm{a}}$, Michela Tosetti ${ }^{\mathrm{g}}$, \\ Riccardo Trinchero $^{\mathrm{h}}$, Christine Wyss $^{\mathrm{j}}$, Sibylle Ziegler ${ }^{\mathrm{c}, \mathrm{p}}$, TRIMAGE Consortium ${ }^{1}$ \\ a Dipartimento di Fisica "E. Fermi”, Università di Pisa, and INFN, Sezione di Pisa, Pisa, Italy \\ ${ }^{\mathrm{b}}$ Weeroc s.a.s., Palaiseau, France \\ ${ }^{\mathrm{c}}$ Nuklearmedinische Klinik und Poliklinik, Klinikum rechts der Isar, Technische Universität München, Munich, Germany \\ d Department of Neuroradiology, Klinikum rechts der Isar, Technische Universität München, Munich, Germany \\ e Neuroimaging Center (TUM-NIC), Klinikum rechts der Isar, Technische Universität München, Munich, Germany \\ ${ }^{\mathrm{f}}$ Forschungszentrum Jülich GmbH, Institute of Neuroscience and Medicine, INM4, Jülich, Germany \\ IRCSS, Stella Maris, Calambrone, Pisa, Italy \\ ${ }^{\mathrm{h}}$ Istituto Nazionale di Fisica Nucleare, Sezione di Torino, Torino, Italy \\ ${ }^{\mathrm{i}}$ Advansid s.r.l., Povo, Trento, Italy \\ ${ }^{\mathbf{j}}$ Department of Psychiatry, Psychotherapy and Psychosomatics, University Hospital of Psychiatry Zurich, University of Zurich, Switzerland \\ ${ }^{\mathrm{k}}$ Department of Psychiatry and Psychotherapy, Psychiatric Services of Aargovia, Switzerland \\ ${ }^{1}$ Technological Educational Institute of Athens, Greece \\ ${ }^{\mathrm{m}}$ Inviscan s.a.s., France \\ ${ }^{n}$ RS2Ds.a.s., Mundolsheim, France \\ o Department of Psychiatry, Psychotherapy and Psychosomatics, Faculty of Medicine, RWTH Aachen University, JARA Brain, Aachen, Germany \\ ${ }^{\mathrm{P}}$ Department of Nuclear Medicine, University Hospital, LMU, Munich, Germany \\ ${ }^{\mathrm{q}}$ Department of Psychiatry, Klinikum rechts der Isar, Technische Universität München, Munich, Germany
}

This Corrigendum for the article 'TRIMAGE: a dedicated trimodality (PET/MR/EEG) imaging tool for schizophrenia' by A. Del Guerra and colleagues, concerns the analyses and results of the F-DOPA PET data assessed in Munich. Particularly, it concerns the section 'Results', subsection '3.1.2. Results of the pilot study: analysis of Munich data' and corresponding Figure 7, as well as parts of the Discussion.

The authors noted that in the analysis of Munich F-DOPA PET data, influx rate constants (Kc) were wrongly calculated. Specifically, in the calculation of the integral used in the Patlak graphical analysis, PET frames were treated as equidistant, which led to higher than normal Kc values. We recalculated the Kc values with the correct integral (taking into account different frame lengths) and ran several control analyses to assess their reliability.

In detail, the corrections affect the following parts of the article:

1) Results, section '3.1.2. Analysis of the Munich data': in the last 2 paragraphs, the sentences "Schizophrenic patients showed .... or other striatal sub-regions." have to be replaced by the following: "Schizophrenic patients showed decreased Kc values in the caudate nucleus

\footnotetext{
DOI of original article: http://dx.doi.org/10.1016/j.eurpsy.2017.11.007

* Corresponding author.

E-mail address: alberto.del.guerra@unipi.it (A. Del Guerra).

1 www.trimage.eu.
} 
$\left(0.0103 \pm 0.001 \mathrm{~min}^{-1}\right)$ compared to healthy controls $\left(0.0132 \pm 0.001 \mathrm{~min}^{-1}\right)(\mathrm{p}<0.001)$ (Figure 7 , upper left). Similarly, a decrease of Kc values was found in the functional executive region (Figure 7, lower left), which largely overlaps with the anatomical region of the caudate, for schizophrenic patients $\left(0.0120 \pm 0.001 \mathrm{~min}^{-1}\right)$ compared to controls $\left(0.0145 \pm 0.001 \mathrm{~min}^{-1}\right)(\mathrm{p}=0.002)$. Additionally, patients showed decreased Kc values in the putamen (Figure 7, center upper part) $\left(0.0131 \pm 0.001 \mathrm{~min}^{-1}\right)$ compared to healthy controls $\left(0.0152 \pm 0.002 \mathrm{~min}^{-1}\right)(\mathrm{p}=0.017)$, and the functional correspondent in the sensorimotor striatum (Figure 7 , center lower part) for patients $\left(0.0119 \pm 0.002 \mathrm{~min}^{-1}\right)$ compared to healthy controls $\left(0.0160 \pm 0.002 \mathrm{~min}^{-1}\right)(\mathrm{p}<0.001)$. Finally, a decrease in Kc values was found for the whole striatum (Figure 7 , right) in patients $\left(0.0120 \pm 0.001 \mathrm{~min}^{-1}\right)$ compared to healthy controls $\left(0.0144 \pm 0.001 \mathrm{~min}{ }^{-1}\right)$ $(p=0.002)$. No differences were found for the accumbens nucleus $(p=0.71)$ and the corresponding functional subdivision, namely the limbic striatum $(\mathrm{p}=0.65)$."

2) Figure 7 of the article has to be replaced by the following new Figure 7 :
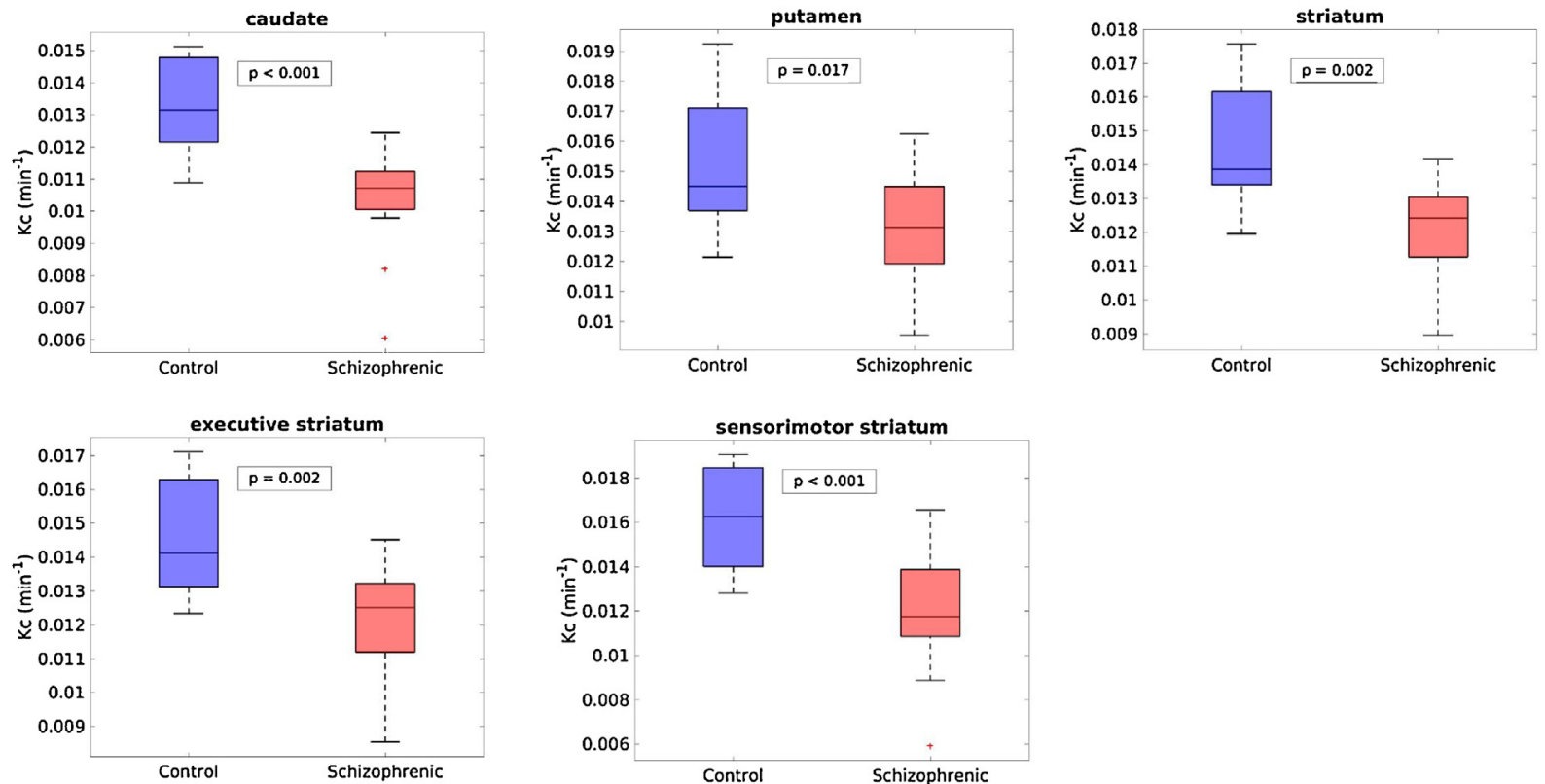

The index of influx, Kc, in the caudate nucleus (upper left), executive region (lower

left), putamen (upper part, center), sensorimotor region (lower part, center), and whole striatum (right) as measured with the PET/MR (mMR) at Munich.

3) In the section '4. Discussion and conclusions', second paragraph, the sentence "The evaluation shows that group differences between healthy controls and schizophrenic patients are well replicable." has to be deleted, since these findings are rather novel and surprising and therefore do not directly replicate previous results. 